\title{
Retinoic acid prevents experimental Cushing syndrome
}

\author{
Marcelo Páez-Pereda, ${ }^{1}$ Damian Kovalovsky, ${ }^{2}$ Ursula Hopfner, ${ }^{1}$ Marily Theodoropoulou, ${ }^{1}$ \\ Uberto Pagotto, ${ }^{1}$ Eberhard Uhl, ${ }^{3}$ Marco Losa, ${ }^{4}$ Johanna Stalla, ${ }^{1}$ Yvonne Grübler, ${ }^{1}$ \\ Cristina Missale, ${ }^{5}$ Eduardo Arzt,${ }^{2}$ and Günter K. Stalla ${ }^{1}$
}

\author{
${ }^{1}$ Max Planck Institute of Psychiatry, Munich, Germany \\ ${ }^{2}$ Laboratorio de Fisiología y Biología Molecular, Universidad de Buenos Aires and Argentine \\ National Research Council (CONICET), Ciudad Universitaria, Buenos Aires, Argentina \\ ${ }^{3}$ Department of Neurosurgery, University of Munich, Munich, Germany \\ ${ }^{4}$ Department of Neurosurgery, San Raffaele Scientific Research Institute (IRCCS) San Raffaele, \\ University of Milano, Milano, Italy \\ ${ }^{5}$ Department of Biomedical Sciences and Biotechnology, University of Brescia, Brescia, Italy \\ Address correspondence to: Marcelo Páez-Pereda, Max Planck \\ Institute of Psychiatry, Kraepelinstrasse 10, 80804 Munich, Germany. \\ Phone: 49-89-30622-272; Fax: 49-89-30622-605; E-mail: marcelo@mpipsykl.mpg.de.
}

Received for publication August 17, 2000, and accepted in revised form August 27, 2001.

Cushing syndrome is caused by an excess of adrenocorticotropic hormone (ACTH) production by neu-
roendocrine tumors, which subsequently results in chronic glucocorticoid excess. We found that
retinoic acid inhibits the transcriptional activity of AP-1 and the orphan receptors Nur77 and Nurr1
in ACTH-secreting tumor cells. Retinoic acid treatment resulted in reduced pro-opiomelanocortin tran-
scription and ACTH production. ACTH inhibition was also observed in human pituitary ACTH-secret-
ing tumor cells and a small-cell lung cancer cell line, but not in normal cells. This correlated with the
expression of the orphan receptor COUP-TFI, which was found in normal corticotrophs but not in
pituitary Cushing tumors. COUP-TFI expression in ACTH-secreting tumor cells blocked retinoic acid
action. Retinoic acid also inhibited cell proliferation and, after prolonged treatment, increased cas-
pase-3 activity and induced cell death in ACTH-secreting cells. In adrenal cortex cells, retinoic acid
inhibited corticosterone production and cell proliferation. The antiproliferative action and the inhi-
bition of ACTH and corticosterone produced by retinoic acid were confirmed in vivo in experimental
ACTH-secreting tumors in nude mice. Thus, we conclude that the effects of retinoic acid combine in
vivo to reverse the endocrine alterations and symptoms observed in experimental Cushing syndrome.
J. Clin. Invest. 108:1123-1131 (2001). DOI:10.1172/JCI200111098.

\section{Introduction}

Cushing syndrome is caused by excessive glucocorticoid secretion due to adrenocorticotropic hormone $(\mathrm{ACTH})$ overproduction by pituitary or lung tumors, or more rarely, to excessive glucocorticoid secretion by adrenal tumors (1-3). This glucocorticoid excess produces abnormal fat deposition, adrenal hyperplasia, thinning of the skin, hypertension, and psychological disturbances. Some inhibitors of ACTH production have been described, but they are not effective in the treatment of Cushing syndrome (4) and so, at the moment, there is no effective pharmacological therapy to control ACTH oversecretion (1-4).

ACTH biosynthesis is coordinately controlled by different transcription factors at the level of the pro-opiomelanocortin (POMC) promoter (5-7). Corticotropinreleasing hormone $(\mathrm{CRH})$, the physiological stimulus for ACTH production, induces c-Fos, Nur77, and Nurr1 expression and CREB, AP-1, and Nur77/Nurr1 transcriptional activity $(6-12)$. AP-1 has been proposed to be involved in POMC transcription (6). However, the main mediators of $\mathrm{CRH}$ are the nuclear orphan receptors Nur77 and Nurr1 (10-12).
Retinoic acid reduces AP-1 and Nur77 binding to their binding sites, resulting in downregulation of AP-1-responsive and Nur77-responsive genes (13-17). On the other hand, retinoic acid has shown antitumorigenic effects (18-21): it inhibits proliferation, invasion, and tumor growth in vivo and induces differentiation and apoptosis (22-25). Some of these effects are due to the inhibition of AP-1 (26). Nur77 is also critical for the antiproliferative effects of retinoic acid on lung cancer cells (27). The retinoic acid response pathways can be inhibited by the orphan receptors COUP-TF (28-30).

Based on these actions of retinoic acid on AP- 1 and Nur77 and on their involvement in ACTH biosynthesis, we examined the effects of retinoic acid on ACTHsecreting tumor cells that cause Cushing syndrome.

\section{Methods}

Cell culture. AtT-20 pituitary corticotroph tumor cells (31) were cultured in DMEM with 2\% FCS, NCI-N592 small-cell lung cancer cells in RPMI-1640 with $10 \%$ FCS, and y1 adrenal cortex tumor cells in Ham-F12 with $15 \%$ horse serum and $2.5 \%$ FCS. ACTH-secreting 

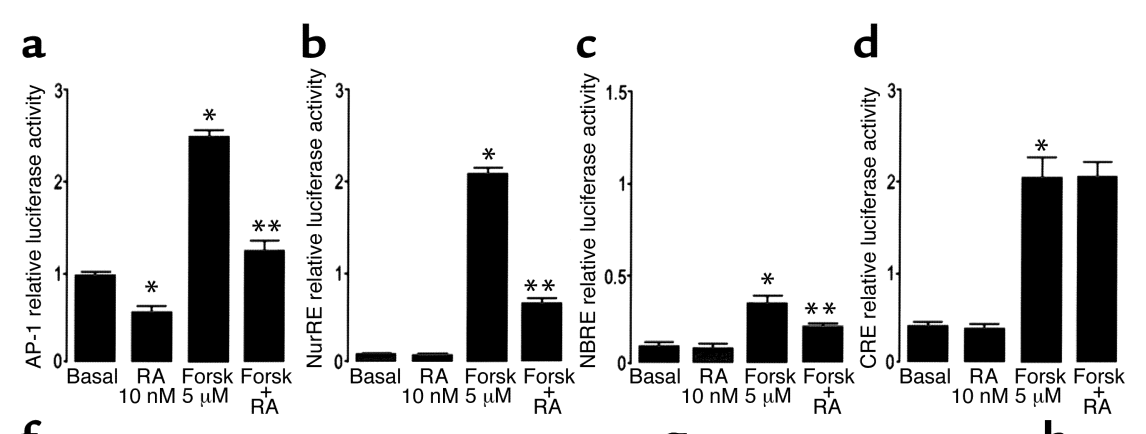

e

\section{f}

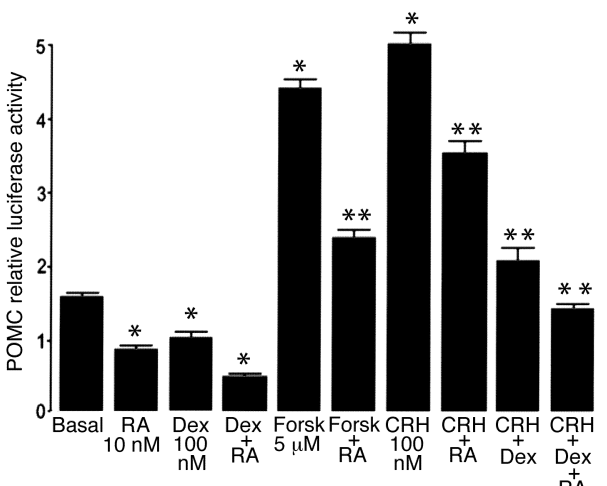

h

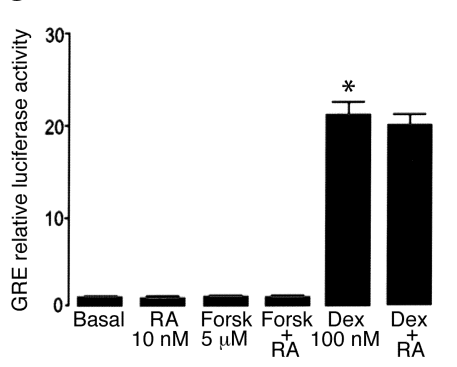

g
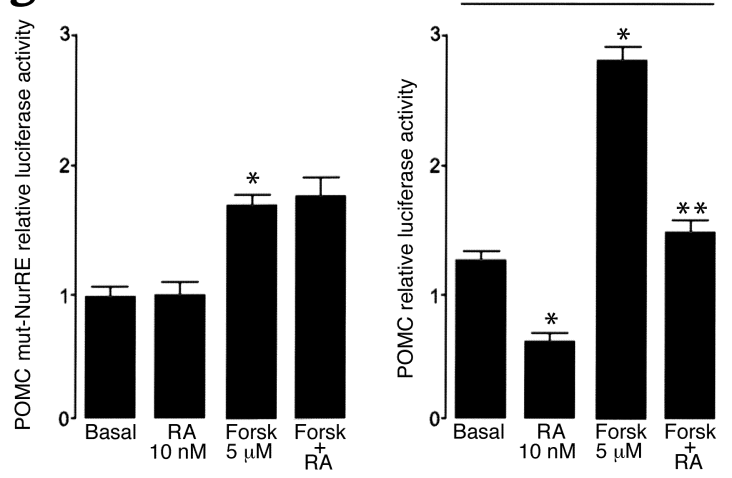

COUP-TFI vector

Figure 1

Retinoic acid inhibits NurRE-directed POMC transcription. AtT-20 cells were transfected with AP-1-LUC (a), NurRE-LUC (b), NBRE-LUC (c), CRE-LUC (d), GRE-LUC (e), POMC-LUC (f), or POMC-mut-NurRE-LUC (g) or cotransfected with POMC-LUC and COUP-TFI expression vector or control vector (h), and treated with $10 \mathrm{nM}$ retinoic acid (RA), $100 \mathrm{nM} \mathrm{CRH,} 100 \mathrm{nM}$ dexamethasone (Dex), or $5 \mu \mathrm{M}$ forskolin (Forsk) or their combinations, as indicated. After a 6-hour treatment, luciferase activity was measured and normalized as described in Methods. Values indicate the mean \pm SE of luciferase-to- $\beta$-galactosidase ratio of one representative experiment out of three, each of them with triplicates of each treatment. ${ }^{*} P<0.01$ compared with the corresponding basal values, ${ }^{*} P<0.01$ compared with CRH or forskolin stimulation, determined by ANOVA in combination with the Scheffé test. Similar results were obtained after a 24-hour treatment with retinoic acid.

pituitary tumors were obtained from patients with Cushing disease. ACTH production in the tumors was confirmed as we described previously (32). Human ACTH-secreting tumor cells and rat normal pituitary cells were cultured in DMEM with 10\% FCS as described previously (32). Only cultures with a vitality higher than $95 \%$ and positive responsiveness to regulatory factors were considered. all-trans-Retinoic acid (Sigma Chemical Co., St. Louis, Missouri, USA) was dissolved in ethanol/DMSO as a $10-\mathrm{mM}$ stock solution and handled in the dark. The same diluting mixture was used in all the in vitro experiments as control.

Hormone measurements. ACTH was determined by RIA as described previously $(33,34)$. Corticosterone was measured by RIA using a ICN Biomedical Inc. (Costa Mesa, California, USA) kit as indicated by the manufacturers.

\section{Figure 2}

Retinoic acid inhibits ACTH production. AtT-20 cells were treated for 2 or 24 hours with 1 or $10 \mathrm{nM}$ retinoic acid, $100 \mathrm{nM} \mathrm{CRH}$, or 1 , 10 , or $100 \mathrm{nM}$ dexamethasone (Dex), or their combinations as indicated. ACTH was measured in the supernatants by RIA. Averages of four wells per treatment and SEs from one representative experiment out of three are shown. ${ }^{*} P<0.01$ compared with the basal values, ${ }^{*} P<0.01$ compared with CRH stimulation, determined by ANOVA in combination with the Scheffé test.
Transfection and reporter assays. Cell transfection was performed with Lipofectamine (Invitrogen Life Technologies, Karlsruhe, Germany). The luciferase gene under the control of $770 \mathrm{bp}$ of the rat POMC promoter includes all the necessary sequences for the expression and regulation of POMC in pituitary cells in vivo (35). The AP-1-LUC construct contains seven repeats of the AP-1 responsive sequence (Stratagene, La Jolla, Califor-

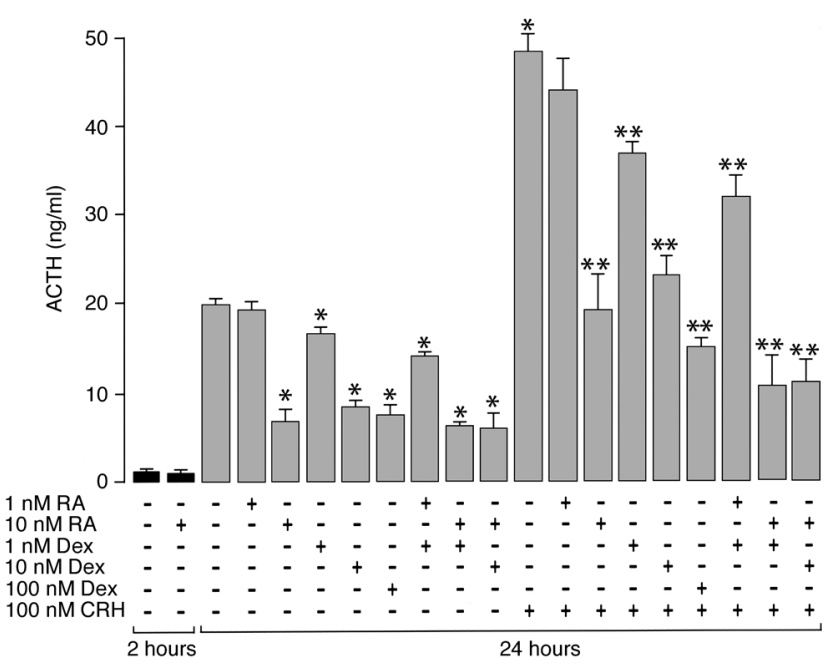


a

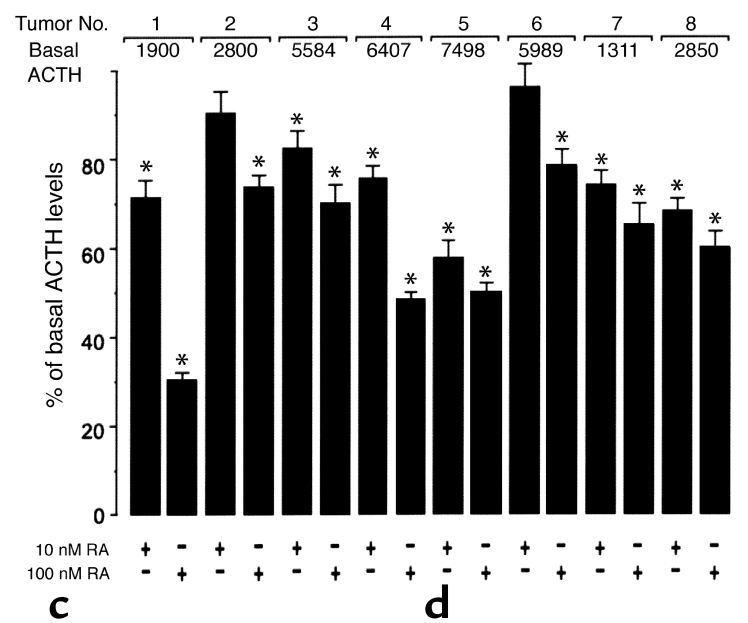

$$
\text { C }
$$
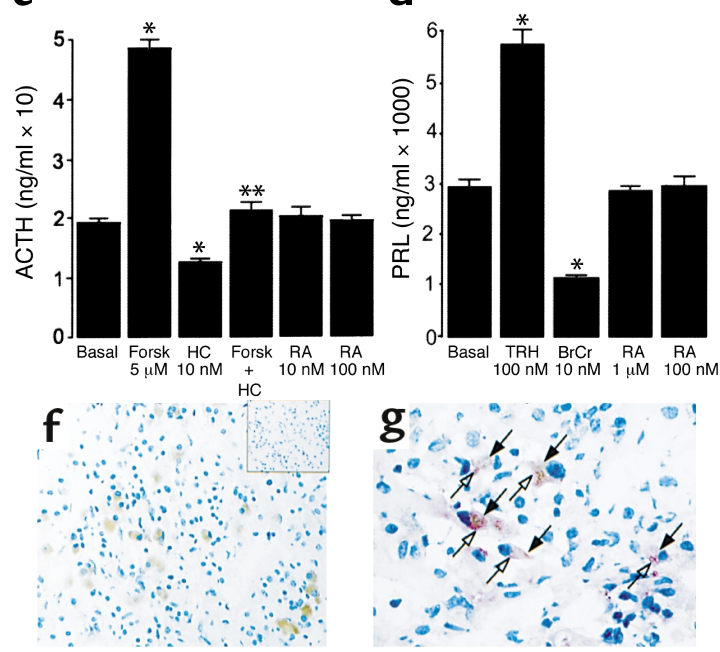

b

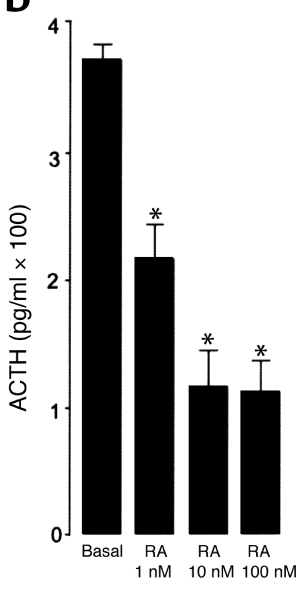

e

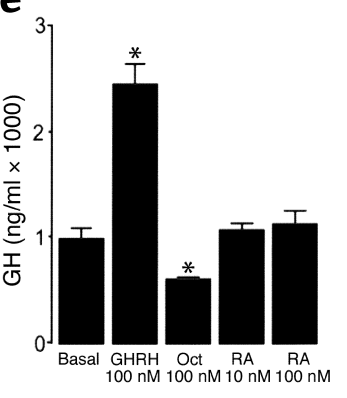

h

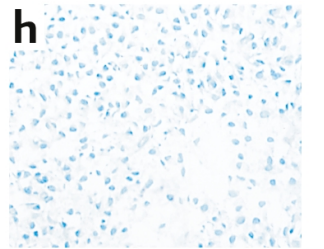

Figure 3

Retinoic acid specifically inhibits ACTH production in human neuroendocrine tumors. (a) Human pituitary adenoma cells from eight patients with Cushing disease were treated with $10 \mathrm{nM}$ or $100 \mathrm{nM}$ retinoic acid (RA) for 24 hours, and ACTH was measured by RIA in the supernatants. Values at the top represent the basal ACTH production of each tumor, and the bars indicate percentages of these values after retinoic acid treatment. (b) NCl-N-592 small-cell lung cancer cells were treated with retinoic acid. After 24 hours, ACTH was measured in the supernatants. (c-e) Normal rat pituitary cells were treated for 24 hours with retinoic acid (RA), $5 \mu \mathrm{M}$ forskolin (Forsk), $10 \mathrm{nM}$ hydrocortisone (HC), 100 $\mathrm{nM}$ thyrotropin-releasing hormone $(\mathrm{TRH}), 1 \mu \mathrm{M}$ bromocriptine $(\mathrm{BrCr}), 100 \mathrm{nM}$ growth hormone-releasing hormone $(\mathrm{GHRH})$, or $100 \mathrm{nM}$ octreotide (Oct). Supernatants were collected and ACTH $(\mathbf{c})$, prolactin $(\mathbf{d})$, and growth hormone $(\mathrm{GH})(\mathbf{e})$ were measured by RIA. Averages of four wells per treatment and the corresponding SEs are shown. ${ }^{*} P<0.001$ compared with the corresponding basal values, ${ }^{*} P<0.01$ compared with the stimulated values, determined by ANOVA in combination with the Scheffé test. $\mathbf{b}-\mathbf{e}$ are representative of three independent experiments. (f) COUP-TFI expression was detected in normal human pituitary $(n=3)$ by immunoperoxidase staining (diaminobenzidine brown color). Inset: negative control. (g) Double immunohistochemistry for COUP-TFI (brown color, filled arrows) and ACTH (vector red color, open arrows). (h) COUP-TFI immunohistochemistry in Cushing adenomas was negative $(n=7)$.

nia, USA). The NurRE-LUC and NBRE-LUC constructs contain three copies of the NurRE or NBRE coupled to the minimal POMC promoter, $-34 /+63$, and the complete POMC promoter contains a mutant NurRE (10, 11). The MTV-LUC construct contains four repeats of the glucocorticoid-responsive element as we described previously (36). Four hundred nanograms of the COUPTFI expression vector containing $1.5 \mathrm{~kb}$ of mouse COUP-TFI cDNA under the control of a CMV promoter or an empty vector was used for the cotransfection experiments (37). The cells were transfected for 6 hours with $500 \mathrm{ng}$ of each plasmid, then incubated for 18 hours and treated for further 6 or 24 hours. Two hun- dred nanograms of the RSV- $\beta$-gal construct was cotransfected in all the experiments to correct for variations in transfection efficiency as described previously $(7,36)$. Luciferase activity was measured with a Berthold luminometer. Results are ratios of luciferase and $\beta$-galactosidase activity. Protein content was measured by a Bradford assay as a further control for the reproducibility of the sampling procedure.

Immunohistochemistry. COUP-TFI was detected using a goat polyclonal (T-19; Santa Cruz Biotechnology, Santa Cruz, California, USA) in combination with anti-goat biotinylated secondary $\mathrm{Ab}$ and avidin-biotinperoxidase complex with diaminobenzidine (Vector 
a

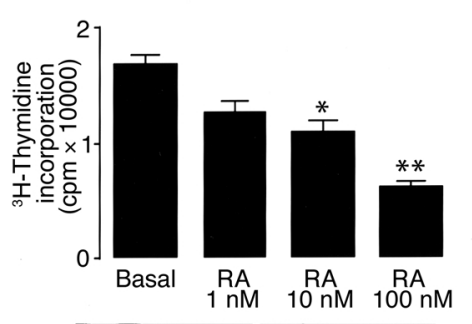

b

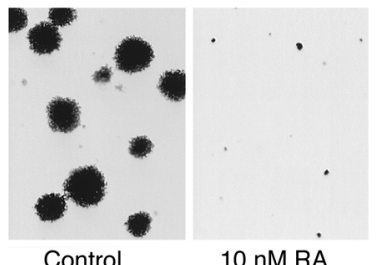

Control

10 nM RA

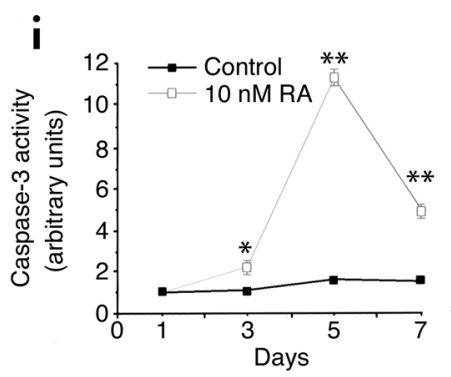

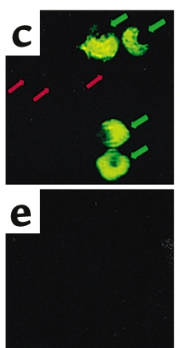

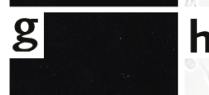

h

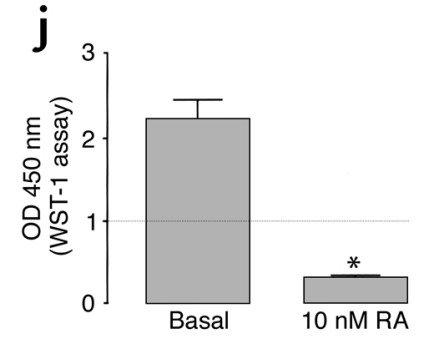

Figure 4

Retinoic acid inhibits proliferation and induces apoptosis (a) AtT-20 cells were treated for 3 days with retinoic acid (RA). Proliferation was measured by ${ }^{3} \mathrm{H}$-thymidine incorporation. (b) AtT-20 cells were treated for 24 hours with 10 $\mathrm{nM}$ retinoic acid and seeded in soft agar. After 20 days, the colonies were stained with MTT and photographed. The control shows colonies composed of more than 300 cells. Retinoic acid-treated cells formed colonies composed of less than ten viable cells. (c-h) AtT-20 cells were stained with annexin V-FITC and photographed under ultraviolet (c, $\mathbf{e}$, and $\mathbf{g}$ ) or white light (d, $\mathbf{f}$, and $\mathbf{h})$. (c and $\mathbf{d}$ ) Cells were treated with $1 \mu \mathrm{M} \mathrm{H}_{2} \mathrm{O}_{2}$ as a positive control for annexin staining induced by apoptosis. Cells were treated with 10 $\mathrm{nM}$ retinoic acid for 6 hours (e and $\mathbf{f}$ ) or 24 hours ( $g$ and $\mathbf{h}$ ). (i) AtT-20 cells were treated with $10 \mathrm{nM}$ retinoic acid. At different times cells were collected, and their vitality was assessed by acridine orange-ethidium bromide staining. Caspase- 3 activity was measured as indicated in Methods. (j) Cell viability was measured after 7 days using the WST-1 assay. The dotted line indicates the WST-1 values at the beginning of the treatment. Averages of four wells per treatment and SEs from one representative experiment are shown. ${ }^{*} P<0.01,{ }^{*} P<0.001$ compared with the corresponding basal or control values, determined by ANOVA in combination with the Scheffé test. The results are representative of four independent experiments.
Laboratories, Burlingame, California, USA). ACTH was detected with a mouse mAb (Dako Diagnostika, Hamburg, Germany) in combination with anti-mouse IgG and mouse alkaline phosphatase (AP)-anti-AP complex (Sigma, Deisenhofen, Germany) with vector red, according to the manufacturer's instructions.

Cell proliferation and viability. ${ }^{3} \mathrm{H}$-thymidine $(0.1$ $\mu \mathrm{Ci} / \mathrm{ml}$ ) was added to the cultures, and its incorporation was measured as described previously (33). A WST1 assay (Roche Molecular Biochemicals, Basel, Switzerland) was also used to measure cell proliferation and cell viability following the manufacturer's instructions (34). Both methods produced similar results and were validated by total cell count. Acridine orange-ethidium bromide staining was used to rule out toxic effects.

Colony formation. Anchorage-independent colony formation was determined as we described previously (34, 38). The cells were treated for 24 hours, suspended in $0.3 \%$ melted agar, and plated on $0.5 \%$ agar. The cultures were incubated for 20 days and stained with dimethylthiazol-diphenyltetrazolium (MTT) to demonstrate the presence of viable cells under all treatments at the end of the experiment.

Annexin staining. Cells were stained with $1 \mathrm{mg} / \mathrm{ml}$ Annexin V-FITC (MedSystems Diagnostics GmbH, Vienna, Austria) for 10 minutes following standard procedures.

Caspase activity measurement. Caspase-3 activity (39) was measured by the proteolytic release of a chromogen from a specific caspase-3 substrate following the manufacturer's instructions (R\&D Systems Inc., Minneapolis, Minnesota, USA). The chromogen was quantified by an ELISA plate reader at $405 \mathrm{~nm}$. The results were normalized to equal amounts of living cells counted by acridine orange-ethidium bromide staining.

Tumor formation and in vivo treatments. ACTH-secreting experimental tumors were formed by injecting AtT-20 cells into a subdermal pouch in BALB/c AnN Crl-nu $\mathrm{BR}$ nude mice (31). To evaluate retinoic acid effects on tumor formation, cells that had been treated with retinoic acid for 3 hours in vitro were trypsinized, checked for vitality, and injected into nude mice. Tumor formation was monitored after 2 and 5 weeks. In a separate set of experiments, mice with 2 -week-old tumors were treated with intraperitoneal injections of $10 \mathrm{mg} / \mathrm{kg}$ retinoic acid dissolved in peanut oil. The treatment was repeated every 4 days for 3 weeks. The tumor volume was measured as we described previously (38). Blood samples were collected by heart puncture from halothane-anesthetized animals. Histological examination was performed by hematoxylin-eosin staining of shock-frozen tissues. All animal experiments were performed in accordance with institutional regulations of animal care.

Statistical analysis. Statistics were performed using onefactor ANOVA in combination with the Scheffé test.

\section{Results}

Retinoic acid effects on ACTH-secreting cells. Basal and forskolin-stimulated AP-1 transcriptional activity were inhibited by retinoic acid in AtT-20 cells after a 6-hour treatment (Figure 1a). Forskolin-stimulated NurREand NBRE-directed transcription, which depend on Nur77 and Nurr1 dimers or monomers, respectively, were also inhibited by retinoic acid (Figure 1, b and c). 
Moreover, retinoic acid inhibited the complete POMC promoter after 6 hours under basal conditions and forskolin or CRH stimulation (Figure 1f). Using a POMC promoter with a mutant NurRE, which is less responsive to forskolin (10-12), we observed that retinoic acid effect was abolished (Figure $1 \mathrm{~g}$ ). Similar results were obtained with all the constructs after a 24 hour retinoic acid treatment (not shown). These results demonstrate that the inhibition of the POMC promoter produced by retinoic acid is mediated by Nur77/Nurr1. The expression of $c$-Fos and Ptx-1 detected by Northern blot analysis was not affected, demonstrating that retinoic acid does not produce a general inhibition of transcription (not shown). Moreover, the transcriptional activities of the glucocorticoid receptor (GR) and CREB, detected by MTV-LUC and CRE-LUC reporters, respectively, were not affected by retinoic acid under basal or stimulated conditions, demonstrating that the inhibition of AP-1-directed and Nur77/Nurr1directed transcription is specific (Figure 1, $\mathrm{d}$ and e). The retinoic acid inhibition of the POMC-LUC reporter activity was blocked by COUP-TFI expression (Figure $1 \mathrm{~h})$. Retinoic acid inhibited not only reporter constructs but also the endogenous ACTH production, which was only observed after 24 hours, suggesting that retinoic acid affects biosynthesis but not ACTH liberation (Figure 2). The inhibition of ACTH produced by dexamethasone was not affected by retinoic acid, and the combined effect of submaximal doses of both substances was additive (Figure 2), in agreement with the effects on POMC transcription (Figure 1f). This suggests that there is no interplay between retinoic acid and the physiological glucocorticoidmediated inhibition of ACTH. Toxic or proliferative effects after a 6- or 24-hour treatment were ruled out by annexin V staining (see Figure 4), acridine orangeethidium bromide staining, and WST-1 assay (not shown). These results demonstrate that retinoic acid specifically inhibits the transcriptional activity of AP-1 and Nur77/Nurr1, thereby reducing POMC transcription and consequently ACTH production.

To evaluate whether retinoic acid would be of profit for human patients we studied the effect on Cushing tumors in primary culture. We observed that $10 \mathrm{nM}$

\footnotetext{
Figure 5

Retinoic acid inhibits corticosterone production and adrenal cortex cell proliferation. (a) y1 adrenal cortex cells were treated for 24 hours with retinoic acid (RA) or $5 \mu \mathrm{M}$ forskolin (Forsk) as indicated. Supernatants were collected and corticosterone was measured by RIA. (b) y1 cells were treated for 3 days with retinoic acid (RA). Proliferation was measured by WST- 1 assay. In $\mathbf{a}$ and $\mathbf{b}$ averages of four wells per treatment and SEs are shown. ${ }^{*} P<0.001$ compared with the forskolin stimulation, determined by ANOVA in combination with the Scheffé test. (c) y1 cells were treated for 24 hours with $10 \mathrm{nM}$ retinoic acid, seeded in soft agar, stained with MTT, and photographed after 20 days. The control shows colonies composed of more than 300 cells. Retinoic acid-treated cells formed colonies composed of less than ten viable cells. These results are representative of three independent experiments with triplicates of each treatment.
}

retinoic acid inhibited ACTH production after a 24hour treatment in six out of eight tumors (Figure 3a). The two other tumors only showed an inhibitory response with $100 \mathrm{nM}$ retinoic acid (Figure 3a; tumor numbers 2 and 6). We also studied the small-cell lung cancer cell line NCI-N592 and found that retinoic acid inhibited ACTH production (Figure $3 \mathrm{~b}$ ). This demonstrates that retinoic acid affects ACTH biosynthesis in different neuroendocrine tumor types. We found that retinoic acid does not affect $\mathrm{ACTH}$, prolactin, or growth hormone production in rat normal pituitary cells, which were responsive to regulatory factors (Figures 3 , $c-e)$. Therefore, we conclude that retinoic acid specifically inhibits ACTH production in ACTH-secreting tumor cells. We found that COUP-TFI, which can block retinoic acid action in ACTH-secreting tumor cells (Figure $1 \mathrm{~h})$, is expressed only in the normal pituitary $(n=3)$ where it colocalizes with ACTH immunoreactivity, but it is not expressed in Cushing pituitary tumors $(n=7)$ (Figure 3, f-h). The same expression pattern of COUPTFI was observed using RT-PCR (not shown).

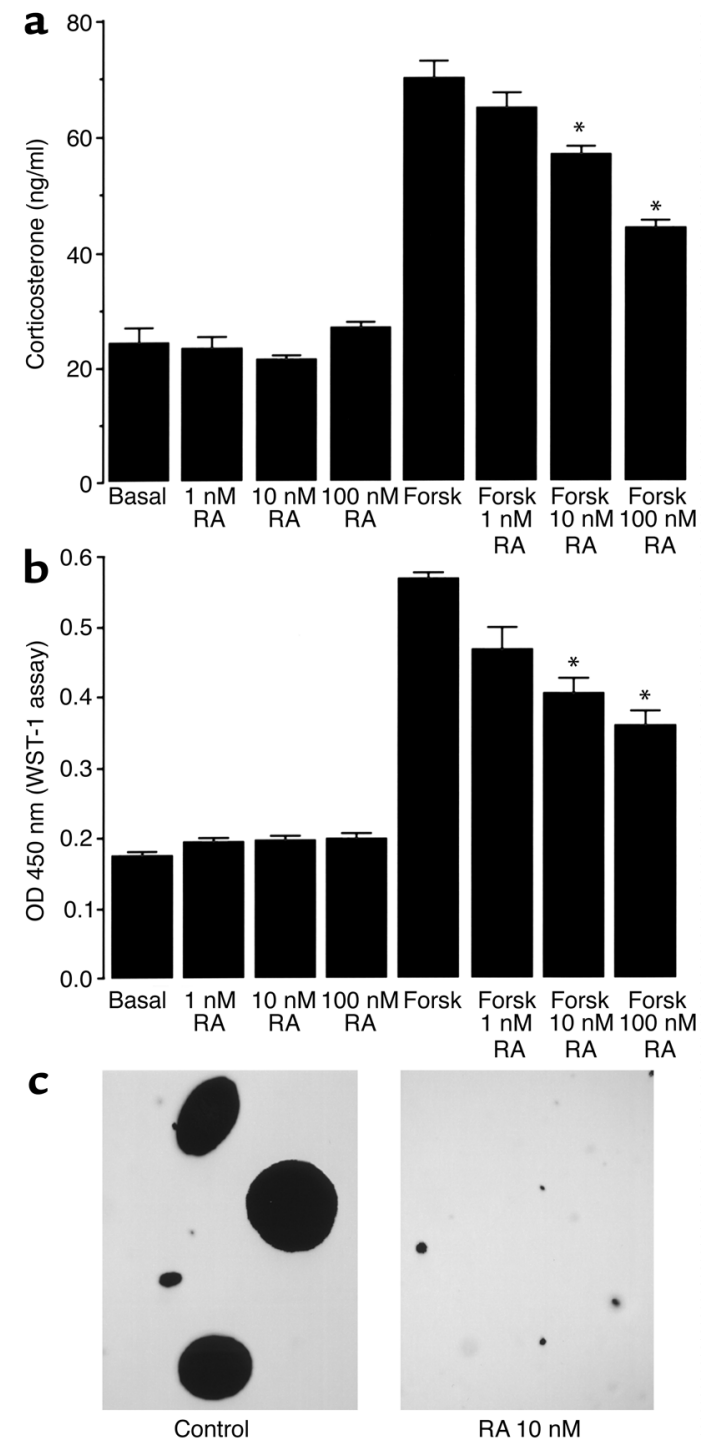



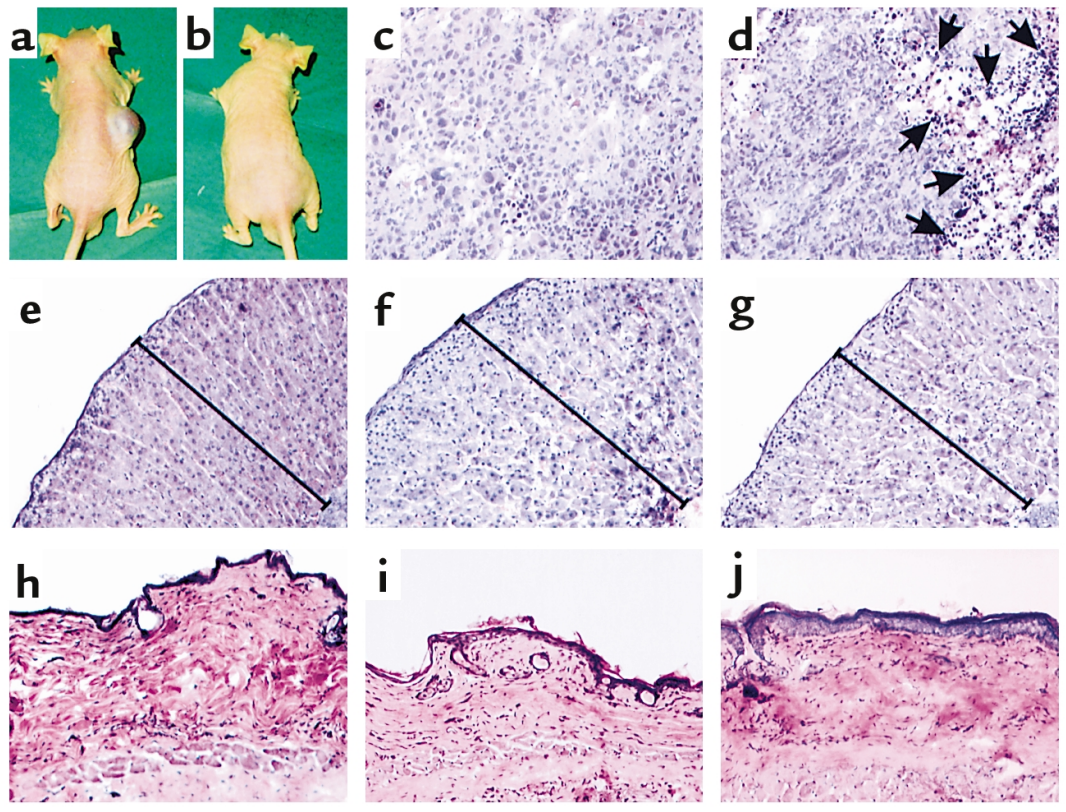

\section{Figure 6}

Retinoic acid inhibits tumor formation and the symptoms of Cushing syndrome. AtT-20 cells were treated with vehicle (a) or $10 \mathrm{nM}$ retinoic acid (b) for 3 hours and then injected into nude mice. ( $\mathbf{a}$ and $\mathbf{b}$ ) Representative examples of the mice after 5 weeks ( $n=10$ in each treatment). Notice the 12-mm-diameter tumor developed by vehicle-treated cells (a). Thinning of the skin was consistently observed in mice with tumors $(\mathbf{a}) .(\mathbf{c}-\mathbf{j})$ Mice with tumors were injected intraperitoneally with $10 \mathrm{mg} / \mathrm{kg}$ retinoic acid or vehicle $(n=5$ in each experiment) every 4 days for 3 weeks. At the end of the treatment the tumors ( $\mathbf{c}$ and $\mathbf{d}$ ) (arrows indicate areas of necrosis), adrenal glands $(\mathbf{e}-\mathbf{g})$, and skin samples $(\mathbf{h}-\mathbf{j})$ were shock-frozen and analyzed by hematoxylin-eosin staining. Adrenals (e) and skin (h) from vehicle-treated mice without tumors. Tumor (c), adrenals (f), and skin (i) from vehicle-treated mice with tumors. Tumor (d), adrenals ( $\mathbf{g}$ ), and skin (j) from retinoic acid-treated mice with tumors. These results are representative of two independent experiments.

To further characterize the actions of retinoic acid we measured cell proliferation. We observed an inhibition of AtT-20 cell proliferation measured by ${ }^{3} \mathrm{H}$-thymidine incorporation after 3 days of retinoic acid treatment (Figure 4a). Similar results were obtained by the WST- 1 assay, and total cell count (not shown) and acridine orange-ethidium bromide staining showed no differences, indicating that there was no cell death. A 24hour treatment with retinoic acid also inhibited anchorage-independent colony formation (Figure 4b), indicating that retinoic acid affects the intrinsic mechanisms controlling cell proliferation.

Retinoic acid did not induce apoptosis after 6- or 24hour treatments as evidenced by annexin $\mathrm{V}$ staining (Figure 4, c-h). We then studied the induction of apoptosis in AtT-20 cells after prolonged retinoic acid treatment by measuring caspase- 3 , a key mediator of the proteolytic cascade leading to apoptosis (39). We observed a marked increase in caspase- 3 activity after 5 days of treatment (Figure 4i). A subsequent reduction in the number of viable cells was observed after 7 days (Figure 4j). The increase in caspase- 3 after 3 days could partially explain the reduction in thymidine incorporation (Figure 4a), although no cell death was observed at that time. These data demonstrate that prolonged retinoic acid treatment induces apoptosis and therefore reduces the number of viable AtT- 20 cells.

Effects of retinoic acid on adrenal cortex cells. We studied the effects of retinoic acid on corticosterone production by adrenal cortex cells. Retinoic acid did not change the basal corticosterone production in y1 adrenal cortex cells after a 24hour treatment, but partially inhibited the stimulation produced by forskolin, which mimics ACTH effects (Figure 5a). We ruled out toxic or proliferative effects by acridine orange-ethidium bromide staining and WST-1 assay. We also found that retinoic acid did not have any effect on the basal cell proliferation but inhibited the stimulation produced by forskolin after 3 days (Figure $5 b$ ). No differences in cell viability were observed after prolonged treatment (not shown). Retinoic acid also inhibited the anchorage-independent colony formation (Figure 5c). Therefore, we conclude that retinoic acid inhibits the forskolin-stimulated corticosterone secretion and cell proliferation in adrenal cortex cells.

In vivo effects of retinoic acid. To test whether retinoic acid affects the ability of ACTH-secreting cells to form tumors in vivo we treated AtT-20 cells for 3 hours with retinoic acid and then injected them into nude mice. After 2 weeks, all the mice injected with vehicletreated cells developed tumors, whereas no tumors were observed in the mice injected with retinoic acid-treated cells. After 5 weeks all mice injected with vehicle-treated cells had a marked thinning of the skin, enlarged adrenal glands, and adrenal cortex hyperplasia (Figure 6, a and f, as compared with Figure 6e and Figure 7b). The plasma levels of ACTH and corticosterone were greatly elevated (Figure 7, $\mathrm{c}$ and d). These symptoms mimic Cushing syndrome in humans. Only $20 \%$ of the mice injected with retinoic acid-treated cells developed small tumors after 5 weeks. The mice injected with retinoic acid-treated cells that did not develop tumors did not show any noticeable changes (Figure $6 \mathrm{~b})$. Therefore, we deduce that retinoic acid pretreatment reduces the ability of ACTH-secreting cells to form tumors in vivo.

To test the efficacy of retinoic acid treatment on tumor growth after implantation, we administered retinoic acid in vivo to mice that already had experimental ACTH-secreting tumors. Specifically, we injected mice that had 2-week-old tumors with $10 \mathrm{mg} / \mathrm{kg}$ retinoic acid intraperitoneally every 4 days for 3 weeks. 
The tumor volume was monitored throughout the treatment. Retinoic acid completely blocked the growth of corticotroph tumors (Figure 7a). Histological examination showed that the tumors under retinoic acid treatment had signs of focal cell death (Figure 6, d as compared with c). These results demonstrate that in vivo treatment with retinoic acid inhibits corticotroph tumor growth and supports our in vitro findings on cell proliferation and apoptosis. We found that retinoic acid partially but significantly reduces plasma ACTH levels (Figure 7c). Corticosterone was reduced by retinoic acid to levels similar to the control (Figure 7d). Therefore, retinoic acid administered in vivo reverses the endocrine alterations produced by corticotroph tumors. This is in agreement with the effects we observed in vitro in ACTH- and corticosterone-secreting cells. Adrenal hyperplasia induced by the tumors was prevented by retinoic acid (Figure $7 \mathrm{~b}$ ). This was also observed in histological sections (Figure 6, g as compared with $\mathrm{f}$ ). The dermal atrophy induced by the ACTH-secreting tumors was also prevented by retinoic acid (Figure $6, j$ as compared with i). All these results indicate that retinoic acid effectively reduces in vivo symptoms of Cushing syndrome.

\section{Discussion}

We found that retinoic acid inhibits the AP-1 and Nur77/Nurr1 transcriptional activities in ACTH-secreting cells. These results are in line with previous studies on other cells that demonstrate that retinoic acid can inhibit AP-1-dependent genes (13-16) and also represses Nur77 transactivation activity (17). We further demonstrate that retinoic acid inhibits POMC transcription, which is controlled by AP-1, Nur77, and Nurr1 (5-12). Moreover, we demonstrate that a mutation in the Nur77/Nurr1-binding site NurRE is sufficient to abolish the inhibition produced by retinoic acid. This inhibition is similar to the inhibition of POMC transcription produced by mutation of the NurRE or AP-1-binding sites (5). The stimulation of POMC by $\mathrm{CRH}$, which is mediated by Nur77 and could also involve AP-1, was inhibited by retinoic acid. These results are also in agreement with the reduction of the $\mathrm{CRH}$-stimulated POMC transcription produced by the mutation of the NurRE or AP-1-binding sites $(6,10)$. The NBRE-binding site, which, as previously shown, is less sensitive to stimulation, is slightly inhibited by retinoic acid. Therefore, it is less relevant than the NurRE site for retinoic acid effects. On the other hand, factors that are important for the function of corticotroph cells such as CREB, GR, and Ptx- 1 were not affected by retinoic acid, demonstrating the specificity of the effects we observed. Our results were confirmed by the fact that the endogenous production of ACTH was reduced by retinoic acid in AtT-20 cells. ACTH inhibition was observed in tumor cells with pituitary or lung origin, demonstrating that the ACTH biosynthesis is affected by retinoic acid in different tumor types. However, ACTH inhibition was observed only in tumor cells but not in normal pituitary cells. Interestingly, this effect is different from the one of glucocorticoids, which efficiently inhibit ACTH production in normal corticotroph cells but have only a partial effect on tumors. This partial insensitivity to glucocorticoids makes Cushing tumors resistant to the physiologic negative feedback. Therefore, retinoic acid does not constitute a physiological feedback mechanism, but the profile of retinoic acid effects makes it even more suitable as a possible therapy for Cushing syndrome. We found that COUP-TFI, which inhibits the retinoic acid response pathways (28-30), can also block the effects of retinoic acid in ACTH-secreting tumor cells. Moreover, COUP-TFI is only expressed in normal ACTH-secreting cells but not in pituitary Cushing tumors. This expression pattern may provide an explanation for the differential effects of retinoic acid on tumor cells. Other genes that have been found to be differentially expressed in pituitary tumors might also be involved in the differential action of retinoic acid (40).
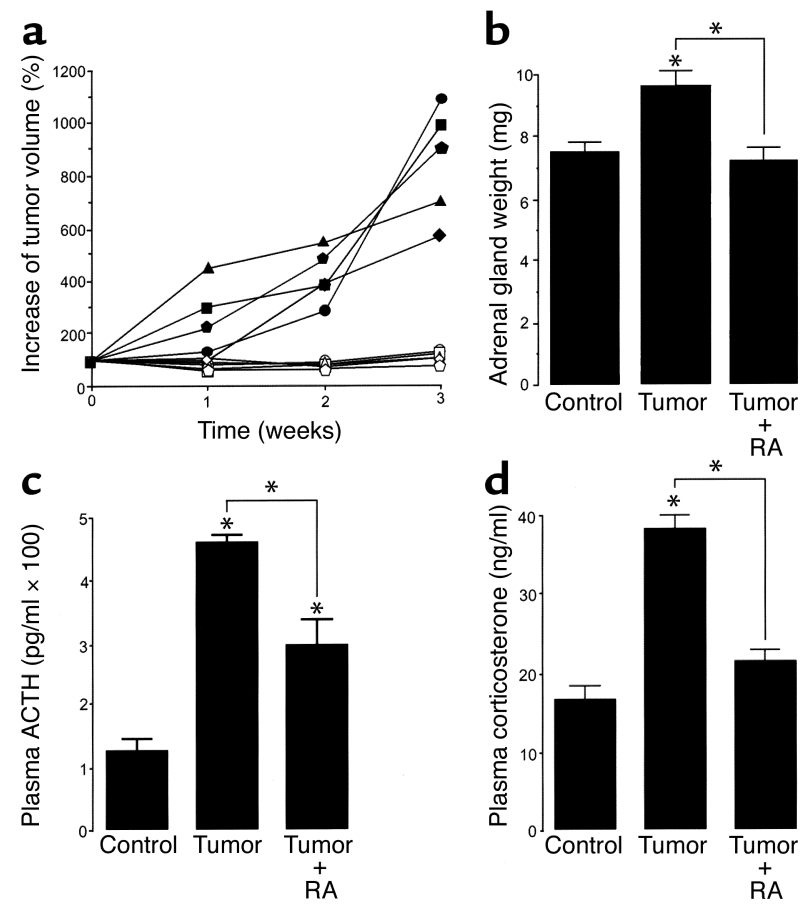

Figure 7

Retinoic acid inhibits tumor growth and endocrine alterations. (a) Mice with 2-week-old tumors formed with untreated AtT-20 cells were injected intraperitoneally with $10 \mathrm{mg} / \mathrm{kg}$ retinoic acid (open symbols, RA) or vehicle (filled symbols) every 4 days for 3 weeks. Tumor volume was monitored during this period. Results show the increase in percentage over the initial tumor volume. Each curve represents a single tumor. (b) At the end of the treatment the weight of the adrenal glands was measured in mice without tumors (control, $n=5)$ and in mice with tumors with or without retinoic acid treatment (RA) as indicated ( $n=5$ for each treatment). (c and d) At the end of the treatment blood was collected and ACTH and corticosterone were measured by RIA. Values indicate the mean \pm SE of one experiment. ${ }^{*} P<0.01$, as determined by ANOVA in combination with the Scheffé test. The results are representative of two independent experiments. 
We found that retinoic acid inhibits ACTH- and corticosterone-secreting cell proliferation. At least some of the antiproliferative effects of retinoic acid are due to the inhibition of AP-1-dependent genes (26). Interestingly, the balance between Nur77 and COUP-TF is also critical for the antiproliferative effects of retinoic acid in lung cancer (27). Due to the fact that retinoic acid has pleiotropic effects, we cannot exclude the possibility that other pathways independent of AP-1 or Nur77/Nurr1 could contribute to the antiproliferative effects. However, the end effect of retinoic acid on ACTH- and corticosterone-secreting tumor cells is antiproliferative in vivo and in vitro. Moreover, the induction of caspase- 3 activity and the subsequent reduction in cell viability, together with the induction of focal cell death in vivo, contribute to the antitumorigenic effect in ACTH-secreting cells.

The effects of retinoic acid in vitro are paralleled by the effects we observed in vivo, and furthermore they cooperate to suppress the symptoms of Cushing syndrome. Retinoic acid inhibition of proliferation and induction of apoptosis/cell death combine to reduce the tumor mass. The inhibition of ACTH and corticosterone secretion together with the reduction of tumor mass cooperate to reverse the endocrine alterations produced by Cushing tumors in vivo, resulting in the reversal of such symptoms of Cushing syndrome as adrenal hyperplasia and skin atrophy. The reduction of adrenal hyperplasia could be due to the reduction in ACTH levels as well as to the direct inhibition of adrenal cell proliferation. The reversion of skin atrophy is in line with previous studies that show that retinoic acid can reverse the changes produced by glucocorticoids on dermal connective tissue (41). Therefore, retinoic acid could reverse the symptoms in the skin by a direct mechanism that may concur with the hormonal inhibition and the antitumorigenic effects.

Clinical studies have shown that retinoids are effective in the treatment of acute promyelocytic leukemia and squamous cell carcinomas of the skin and cervix and in the chemoprevention of lung cancer (18-21). The doses of retinoic acid used in our study are within the dose range currently used to treat patients with various neoplasias (18-21). On the other hand, retinoic acid has been shown to stimulate growth hormone in vitro in the lacto-somatotrophic tumor cell line GH3 (42). In our study we have not detected changes in growth hormone or prolactin production in normal pituitary cells. Therefore, the differential effect of retinoic acid on ACTH could, hypothetically, represent a therapeutic advantage. The immunostimulation produced by retinoic acid (43) could also be of benefit for Cushing patients who show some impaired immune functions due to glucocorticoid excess. These characteristics of retinoic acid further support the possibility of a successful treatment of ACTH-secreting tumors in human patients, for whom there is no effective pharmacological therapy.

To summarize, we describe the specific inhibitory effects of retinoic acid on ACTH biosynthesis at the level of AP-1 and Nur77/Nurr1 transcriptional activities and POMC transcription in ACTH-secreting tumor cells. We demonstrate that retinoic acid reduces cell proliferation and viability. These results are also supported by the inhibition of hormone production and tumor growth observed in vivo. All these results indicate that retinoic acid might be an interesting therapeutic option to inhibit ACTH, cortisol, and tumor growth in Cushing syndrome.

\section{Acknowledgments}

We thank M. Low (Vollum Institute, Portland, Oregon, USA) for the POMC-LUC construct, J. Drouin (University of Montréal, Quebec, Canada) for the NurRE and NBRE reporters and the POMC promoter with a mutated NurRE site, and M. Tsai (Baylor College of Medicine, Houston, Texas, USA) for the COUP-TFI expression vector. We also thank W. Bollag (Roche, Basel, Switzerland) and O. Almeida (Max Planck Institute of Psychiatry, Munich, Germany) for comments on the manuscript. This work was supported by the Deutsche Forschungsgemeinschaft (Sta 285/7-3) and the Volkswagen Foundation (I/76 803).

1. Orth, D.N. 1995. Cushing's syndrome. N. Engl. J. Med. 332:791-803.

2. Dahia, P.L.M., and Grossman, A. 1999. The molecular pathogenesis of corticotroph tumors. Endocr. Rev. 20:136-155.

3. Wajchenberg, B.L., et al. 1994. Ectopic adrenocorticotropic hormone syndrome. Endocr. Rev. 15:752-787.

4. Engler, D., Redei, E., and Kola, I. 1999. The corticotropin-release inhibitory factor hypothesis: a review of the evidence for the existence of inhibitory as well as stimulatory hypophysiotropic regulation of adrenocorticotropin secretion and biosynthesis. Endocr. Rev. 20:460-500.

5. Therrien, M., and Drouin, J. 1991. Pituitary pro-opiomelanocortin gene expression requires synergistic interactions of several regulatory elements. Mol. Cell. Biol. 11:3492-3503.

6. Boutillier, A.L., et al. 1995. Corticotropin-releasing hormone stimulates proopiomelanocortin transcription by cFos-dependent and -independent pathways: characterization of an AP-1 site in exon 1. Mol. Endocrinol. 9:745-755.

7. Bousquet, C., Zatelli, M.C., and Melmed, S. 2000. Direct regulation of pituitary proopiomelanocortin by STAT3 provides a novel mechanism for immuno-neuroendocrine interfacing. J. Clin. Invest. 106:1417-1425.

8. Seasholtz, A. 2000. Regulation of adrenocorticotropic hormone secretion: lessons from mice deficient in corticotropin-releasing hormone. J. Clin. Invest. 105:1187-1188.

9. Autelitano, D.J., and Cohen, D.R. 1999. CRF stimulates expression of multiple fos and jun related genes in the AtT-20 corticotroph cell. Mol. Cell. Endocrinol. 119:25-35.

10. Philips, A., et al. 1997. Novel dimeric Nur77 signaling mechanism in endocrine and lymphoid cells. Mol. Cell. Biol. 17:5946-5951.

11. Philips, A., et al. 1997. Antagonism between Nur77 and glucocorticoid receptor for control of transcription. Mol. Cell. Biol. 17:5952-5959.

12. Maira, M., Martens, C., Philips, A., and Drouin, J. 1999. Heterodimerization between members of the Nur subfamily of orphan nuclear receptors as a novel mechanism for gene activation. Mol. Cell. Biol. 19:7549-7557.

13. Schule, R., et al. 1991. Retinoic acid is a negative regulator of AP-1responsive genes. Proc. Natl. Acad. Sci. USA. 88:6092-6096.

14. Pfahl, M. 1993. Nuclear receptor/AP-1 interaction. Endocr. Rev. 14:651-658.

15. Nagpal, S., Athanikar, J., and Chandraratna, R.A. 1995. Separation of transactivation and AP1 antagonism functions of retinoic acid receptor alpha. J. Biol. Chem. 270:923-927.

16. Zhou, X.F., Shen, X.Q., and Shemshedini, L. 1999. Ligand activated retinoic acid receptor inhibits AP-1 transactivation by disrupting cJun/c-Fos dimerization. Mol. Endocrinol. 13:276-285.

17. Kang, H.J., et al. 2000. Retinoic acid and its receptors repress the expression and transactivation functions of Nur77: a possible mechanism for the inhibition of apoptosis by retinoic acid. Exp. Cell Res. 256:545-554.

18. Lotan, R. 1996. Retinoids in cancer chemoprevention. FASEB J. 10:1031-1039. 
19. Hong, W.K., and Sporn, M.B. 1997. Recent advances in chemoprevention of cancer. Science. 278:1073-1077.

20. Kurie, J.M. 1999. The biologic basis for the use of retinoids in cancer prevention and treatment. Curr. Opin. Oncol. 11:497-502.

21. Kelloff, G.J. 2000. Perspectives on cancer chemoprevention research and drug development. Adv. Cancer Res. 78:199-234.

22. Bertram, J.S. 1983. Inhibition of neoplastic transformation in vitro by retinoids. Cancer Surv. 3:243-262.

23. McGarvey, T.W., Silberman, S., and Persky, B. 1990. The effect of butyric acid and retinoic acid on invasion and experimental metastasis of murine melanoma cells. Clin. Exp. Metastasis. 8:433-448.

24. Páez-Pereda, M., et al. 1999. Retinoic acid stimulates meningioma cell adhesion to the extracellular matrix and inhibits invasion. Br. J. Cancer. 81:381-386.

25. Páez-Pereda, M., et al. 2000. Nerve growth factor and retinoic acid inhibit proliferation and invasion in thyroid tumor cells. Mol. Cell. Endocrinol. 167:99-106.

26. Fanjul, A., et al. 1994. A new class of retinoids with selective inhibition of AP-1 inhibits proliferation. Nature. 372:107-111.

27. Wu, Q., et al. 1997. Modulation of retinoic acid sensitivity in lung cancer cells through dynamic balance of orphan receptors nur77 and COUP-TF and their heterodimerization. EMBOJ. 16:1656-1669.

28. Tran, P., et al. 1992. COUP orphan receptors are negative regulators of retinoic acid response pathways. Mol. Cell. Biol. 12:4666-4676.

29. Kliewer, S.A., et al. 1992. Retinoid X receptor-COUP-TF interactions modulate retinoic acid signalling. Proc. Natl. Acad. Sci. USA. 89:1448-1452.

30. Cooney, A.J., Leng, X., Tsai, S.Y., O’Malley, B.W., and Tsai, M.J. 1993. Multiple mechanisms of chicken ovalbumin upstream promoter transcription factor-dependent repression of transactivation by the vitamin $\mathrm{D}$, thyroid hormone, and retinoic acid receptors. J. Biol. Chem. 268:4152-4160.

31. Leung, C.K.H., Paterson, J.A., Imai, Y., and Shiu, R.P.C. 1982. Transplantation of ACTH-secreting pituitary tumor cells in athymic nude mice. Virchows Arch. 396:303-312.

32. Arzt, E., et al. 1992. Interleukin-2 and interleukin-2 receptor expression in human corticotrophic adenoma and murine pituitary cell cultures. $J$. Clin. Invest. 90:1944-1951.
33. Arzt, E., et al. 1993. Interleukin involvement in anterior pituitary cell growth regulation: effects of IL-2 and IL-6. Endocrinology. 132:459-467.

34. Páez-Pereda, M., et al. 2000. High levels of matrix metalloproteinase activity regulate proliferation and hormone secretion in pituitary cells. J. Clin. Endocrinol. Metab. 85:263-269.

35. Liu, B., Hammer, G.D., Rubinstein, M., Mortrud, M., and Low, M.J. 1992. Identification of DNA elements cooperatively activating proopiomelanocortin gene expression in the pituitary glands of transgenic mice. Mol. Cell. Biol. 12:3978-3990.

36. Costas, M., et al. 1996. Molecular and functional evidence for in vitro cytokine enhancement of human and murine target cell sensitivity to glucocorticoids. TNF-alpha priming increases glucocorticoid inhibition of TNF-alpha-induced cytotoxicity/apoptosis. J. Clin. Invest. 98:1409-1416.

37. Pipaon, C., Tsai, S.Y., and Tsai, M.-J. 1999. COUP-TF upregulates NGFIA gene expression through an Sp1 binding site. Mol. Cell. Biol. 19:2734-2745.

38. Missale, C., et al. 1998. Nerve growth factor abrogates the tumorigenicity of human small cell lung cancer cell lines. Proc. Natl. Acad. Sci. USA. 95:5366-5371.

39. Casciola-Rosen, L., et al. 1996. Apopain/CPP32 cleaves proteins that are essential for cellular repair: a fundamental principle of apoptotic death. J. Exp. Med. 183:1957-1964.

40. Heaney, A.P., Horwitz, G.A., Wang, Z., Singson, R., and Melmed, S. 1999. Early involvement of estrogen-induced pituitary tumor transforming gene and fibroblast growth factor expression in prolactinoma pathogenesis. Nat. Med. 5:1317-1321.

41. Schwartz, E., Mezick, J.A., Gendimenico, G.J., and Kligman, L.H. 1994. In vivo prevention of corticosteroid-induced skin atrophy by tretinoin in the hairless mouse is accompanied by modulation of collagen, glycosaminoglycans, and fibronectin. J. Invest. Dermatol. 102:241-246.

42. Morita, S., Fernandez-Mejia, C., and Melmed, S. 1989. Retinoic acid selectively stimulates growth hormone secretion and messenger ribonucleic acid levels in rat pituitary cells. Endocrinology. 124:2052-2056.

43. Dennert, G. 1985. Immunostimulation by retinoic acid. Ciba Found. Symp. 113:117-131. 